\title{
Optical Feedback Radiation Forces: Intracavity Optical Trapping with Feedback-locked Diode Lasers
}

\author{
Yazgan Tuna $^{1}$, Rania Sayed ${ }^{2,3}$, Maria G. Donato ${ }^{2}$, Pietro G. Gucciardi' ${ }^{2}$, \\ Onofrio M. Maragò ${ }^{2, *}$ and Giovanni Volpe ${ }^{1,+}$ \\ 1. Department of Physics, Bilkent University, Çankaya, Ankara, Turkey \\ 2. CNR-IPCF, Istituto per i Processi Chimico-Fisici, I-98158 Messina, Italy \\ 3. Dottorato in Fisica dell'Università di Messina, Dip. di Fisica, F. S. D'Alcontres 31, 98166 S. Agata-Messina, Italy \\ *marago@me.cnr.it \\ +giovanni.volpe@fen.bilkent.edu.tr
}

\begin{abstract}
We demonstrate a novel mechanism for optical tweezing, where a trapped particle dynamically alters an external cavity quality factor, reduceing the average intensity and photodamage, even employing low-numerical aperture lenses and wide fields-of-view.

OCIS codes: (140.7010) Laser trapping; (170.4520) Optical confinement and manipulation; (120.5820) Scattering measurements; (180.0180) Microscopy.
\end{abstract}

Optical tweezers (OT) are generally realized by highly focusing a laser beam in order to trap a mesoscopic dielectric particle near the focal spot [1]. The resulting radiation forces are typically distinguished in scattering forces, which are due to the light forward pressure, and gradient forces, generated by the gradient of the light intensity. OT have been extensively applied to cellular and molecular biology, soft matter, nanotechnology and nanoscale thermodynamics. One of the issues in using OT with sensitive samples, e.g., living cells and biomolecules, is the fact that they may induce photodamage when the average radiation intensity exceeds some threshold.

Here, we describe a novel approach to optical trapping based on optical feedback-locking. In this novel configuration (see Fig. 1), the optical feedback on a diode laser source is controlled by the light scattering from a trapped particle. When no particle is in the trap, the optical feedback from a dielectric mirror posed above the microscope objective will increase the trapping power in the focal spot. Instead, when a particle falls in the trap the optical feedback will be reduced and trap will work at low power preventing damage and relaxing the stringent conditions on high numerical aperture for standard OT. One of the key advantages is that with this approach it is possible to use low numerical aperture lenses $(\mathrm{NA}=0.45)$ and, therefore, large field of view (in excess of $1000 \mu \mathrm{m} \times 1000 \mu \mathrm{m})$. Furthermore, this technique enables a reduction of the average light intensity employed for a given trap stiffness minimizing the photodamage at the sample.

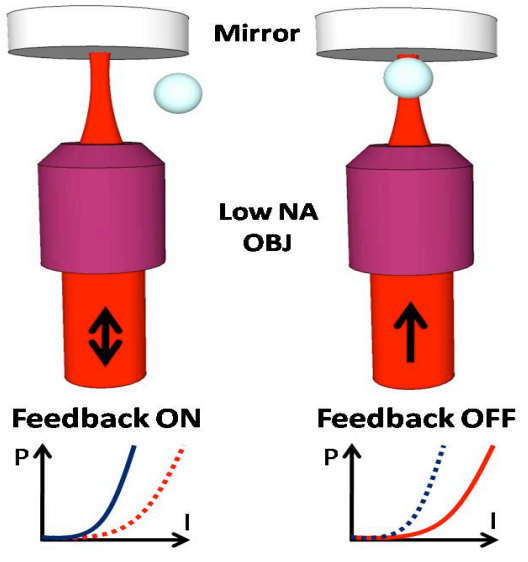

Figure 1. Sketch of the experimental setup and methodology for feedback-controlled optical trapping. (left) When no particle is trapped the optical feedback on the diode laser is on and the available power at the sample permits to efficiently attract particles to the focal spot of the low numerical aperture objective. (right) When a particle is trapped the feedback is off and the trap works at lower power reducing photodamage on the trapped sample.

As laser source, we employ a diode laser, because diode lasers can be built to meet stringent specifications on beam stability, optical beam shape, stable wavelength or small thermal drift, as well as small dimensions and low cost. Since stabilization of laser frequency is essential for various research 
fields such as metrology, frequency standards, and optical communications, the behavior of diode lasers in the presence of optical feedback is well understood [2].

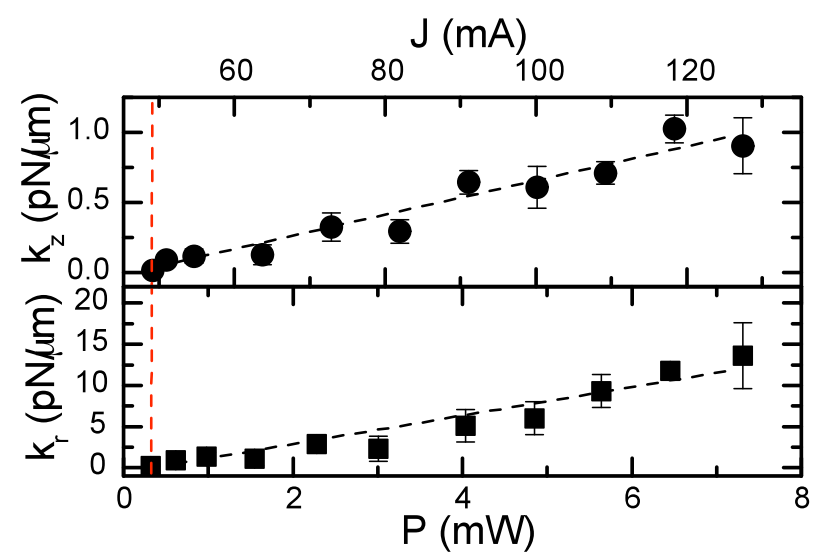

Figure 2. Axial (above) and radial (below) trap stiffness as a function of the laser power (P) and of the diode laser injection current $(J)$ in the presence of optical feedback. The dashed red line corresponds to the threshold current of the free-running operation.

We obtained stable trapping at power as low as $0.32 \mathrm{~mW}$ with an injection current close to the diode laser free-running threshold. This corresponds to an intensity two order of magnitude lower than a standard OT based on a high NA objective lens. When a bead is trapped, we observe a modification of the feedback coupling. This perturbation depends on the scattered field and has a time dependence that is related to the thermal fluctuations of the trapped particle. When these are small, we are in a linear regime, and we can monitor the intracavity intensity fluctuations to calibrate optical forces. Autocorrelation function (ACF) analysis $[3,4]$ is then used to obtain the trap force constants presented in figure 2.

In conclusion, we have demonstrated a novel mechanism for optical trapping that employs a low numerical aperture lens and a mirror to feedback lock a diode laser source. The modification of the intracavity dynamics actively stabilizes the particle trapping. This open perspectives for novel applications of optical trapping to samples that require lower intensity and increased fields of view. Such applications are particularly interesting for dealing with biological samples that are prone to photodamage.

[1] A. Ashkin, "History of Optical Trapping and Manipulation of Small-Neutral Particle, Atoms, and Molecules," IEEE J. Sel. Top. Quant. El. 6, 841-856 (2000).

[2] J. H. Osmundsen and N. Gade, "Influence of Optical Feedback on Laser Frequency Spectrum and Threshold Conditions," IEEE J. Quant. El. 19, 465-469 (1983).

[3] G. Volpe and D. Petrov, “Torque Detection Using Brownian Fluctuations," Phys. Rev. Lett. 97, 210603 (2006).

[4] O. M. Maragò et al., "Femtonewton Force Sensing with Optical Trapped Nanotubes," Nano Lett. 8, 3211-3216 (2008). 